\title{
Gambling and Bipolar disorder- a case report
}

S. Nascimento ${ }^{1}$, I. Caldas ${ }^{1}$, J. Oliveira ${ }^{2}$, J. Reis ${ }^{2}$

${ }^{1}$ Psychiatry trainee at Centro Hospitalar Psiquiátrico de Lisboa, Lisbon, Portugal

${ }^{2}$ Psychiatrist at Centro Hospitalar Psiquiátrico de Lisboa, Lisbon, Portugal Behavioral Addictions Ambulatory Team, CHPL sandrateles@Hotmail.com

\section{Introduction}

$\checkmark$ Bipolar disorder (BD) is a severe and chronic condition with lifetime prevalence rates of up to $6.5 \%$ in the general population.

$\checkmark$ Clinical and epidemiological research suggests that behavioral addictions and related disorders (BARD) have a relationship with bipolar disorder (BD); gambling disorder (GD) and kleptomania, in particular, seem to be the most prevalent among BD patients. Although, the relationship between GD and mood disorders is not, necessarily causal (Quiltye al, 2011).

$\checkmark$ GD is characterized by persistent and maladaptive gambling behavior, whereby individuals engage in frequent and repeated episodes of gambling despite serious adverse consequences (Hodgins etal, 2011).

$\checkmark$ Prevalence of GD was significantly higher (6.3\%) amongst BD subjects as compared to the general population (2.0\%) and major depressed patients $(2.5 \%)$ (Mcintyre et al, 2007).

$\checkmark$ GD affects $0.5-1 \%$ of adults worldwide; the consequences of this behavioral disturbance often entail severe damage to the lives of patients and their families (Kessler, 2008). As substance use disorders (SUDs) , GD presents the phenomena of tolerance, withdrawal and craving; associated with an overlap with respect to etiology, biology, comorbidity, and treatment.

These comorbid conditions were associated with a more severe course of illness, a low probability of recovery as well as an unfavorable course and outcome (Mclintre et al, 2004), $\checkmark$ Males (19.5\%) and females (7.8\%) (Kennedy et al, 2010)

$\checkmark$ Alcohol dependence highest risk for GD in BD patients

$\checkmark$ Shared genetic vulnerability for GD and SUDs (unl et al, 2008)

\section{Case report}

Ms. A., a 61-year-old woman, retired, diagnosed with BD since the age of 24 , characterized by mostly depressive episodes with psychotic symptoms, in remission with olanzapine $20 \mathrm{mg}$ id, lithium $400 \mathrm{id}$, valproate acid $1000 \mathrm{id}$. For the past 6 years, the patient developed a persistent and recurrent problematic gambling behavior (not associated with mood episodes of either polarity). The patient was referred to our outpatient team and a trial of naltrexone, gradually titrated to $50 \mathrm{mg}$, was initiated; the problematic behavior ceased after 1 month of treatment but cravings persisted. After developing a new depressive episode, the patient was started on bupropiom with further improvement.

- Symptoms of GD and gambling behavior in Mania share some similarities - feelings of lack of control over impulsivity.

- Depressive symptoms are also a very common- related to engagement in gambling, attempt to relieve a negative mood.

- Anxiety symptoms engage in gambling activities to reduce arousal states, considered as part of withdrawal-like symptoms. (smith etal, 2011).

- Impulsivity - and compulsive features

- Anhedonia, important role, both as part of withdrawal syndromes and as a relevant factor involved in relapse

- Higher susceptibility to craving phenomena has also been observed in BD (mcelroy etal, 2005)

- $\mathrm{BD}$ and GD patients are both characterized by significant neurocognitive impairment

Evidence from animal model studies hypothesizes that system-related change involving functioning of the Dopamine play a role in impulsive choice, risk-taking behavior and reward (van Enkhuizen et al, 2013); combined with the identification of shared neurobiological dysfunctions- are help guide for future studies in BD-GD subjects and in conceiving a biological rationale for pharmacological interventions.

$\checkmark$ Pharmacological treatment strategies for BD-GD comorbidity are limited with several difficulties in the development of reliable and effective treatment guidelines, however, the phenomenological and clinical overlapped, as well as comorbidity, and the existence of a shared psychopathological framework may help guiding treatment options.

- Lithium is the only drug with a IB level of evidence for comorbid BD-GD

- Valproate anti-craving properties, and evidence of efficacy of valproate with comorbid alcohol and substance abuse

- Topiramate could be useful in GD subgroups characterized by high levels of impulsivity

- Antipsychotics do not have a role in the treatment of GD (potential effectiveness of quetiapine, Risperidone may be a useful adjunctive treatment in comorbid BD-GD patients with predominant obsessive-compulsive symptoms )

- Opioid antagonists better than placedo Nalmefene and Naltrexone efficacy in alcohol dependence/probably good option GD

- Care with antidepressants (exception is bupropion) and amantadine - worsen and precipitate manic episode; also, aripiprazole may be detrimental in BARD.

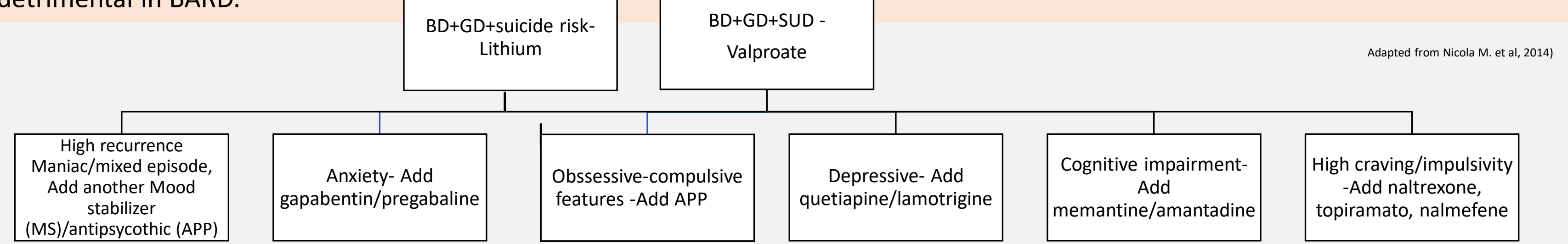

To summarize, BD-GD patients have a more severe course of illness and poorer treatment outcome, associated with a variety of clinical and psychosocial factors that collectively impede remission and recovery. Further trials with long-term follow-up are required to evaluate the efficacy of treatment strategies in large samples (Nicola $\mathrm{M}_{\text {. et al, 2014) }}$ to investigate the differential treatment effects and the potential utility of combined pharmacological and psychosocial treatment strategies (vita et al, 2009) 